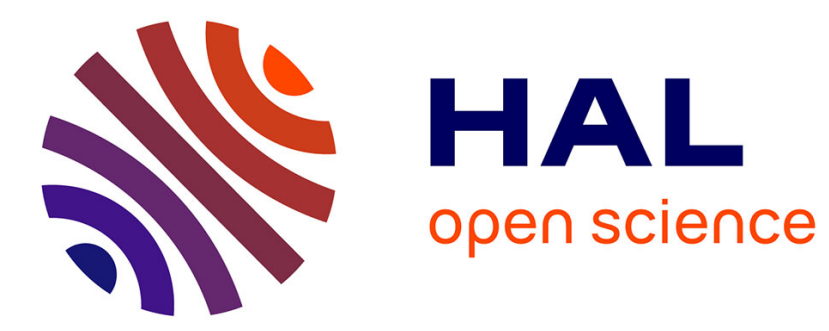

\title{
La collection a-t-elle un avenir au sein du musée ? François Mairesse
}

\section{To cite this version:}

François Mairesse. La collection a-t-elle un avenir au sein du musée ?. Culture et Musées, 2021, 37, pp.31-52. 10.4000/culturemusees.6124 . hal-03279280

\section{HAL Id: hal-03279280 \\ https://hal.science/hal-03279280}

Submitted on 6 Jul 2021

HAL is a multi-disciplinary open access archive for the deposit and dissemination of scientific research documents, whether they are published or not. The documents may come from teaching and research institutions in France or abroad, or from public or private research centers.
L'archive ouverte pluridisciplinaire HAL, est destinée au dépôt et à la diffusion de documents scientifiques de niveau recherche, publiés ou non, émanant des établissements d'enseignement et de recherche français ou étrangers, des laboratoires publics ou privés. 


\section{OpenEdition}

Journals

\section{Culture \& Musées}

Muséologie et recherches sur la culture

$37 \mid 2021$

Les collections patrimoniales ont-elles un avenir?

Dossier

\section{La collection a-t-elle un avenir au sein du musée?}

Do collections have a future in museums?

¿Tiene la colección un futuro dentro del museo?

FrançOIs MaIresSe

p. 31-52

https://doi.org/10.4000/culturemusees.6124

\section{Résumés}

Français English Español

Cet article étudie les conséquences possibles de l'évolution actuelle du phénomène muséal sur la place des collections au sein du musée. La mondialisation, le développement du numérique, ainsi que l'influence de nouveaux courants de pensée, comme le postcolonialisme, pourraient transformer la manière de définir le musée. Cette transformation s'inscrit cependant dans une évolution plus globale, qui se poursuit depuis les années 1970. L'auteur évoque les principaux jalons qui ont conduit les musées à privilégier le public sur la gestion des collections, mais aussi l'émergence de l'autonomie progressive des réserves au sein du système muséal. Ce principe le conduit à envisager de manière conjointe l'évolution des réserves et celle des lieux d'exposition, à la lumière d'autres tendances pouvant être observées au sein du système économique mondial.

This article studies the possible consequences of the current evolution within the museum phenomenon concerning the place of collections within the museum. Globalization, the development of digital technology, and the influence of new currents of thought, such as postcolonialism, could transform the way in which we define museums. However, this change is part of a more global development, which has continued since the 1970s. The author discusses the main trends that have led museums to focus on the public to the detriment of collection management, as well as the emergence of off-site storage within the museums. This principle leads him to consider jointly the evolution of storage and exhibition spaces in light of other trends within the world economic system.

Este artículo examina las posibles consecuencias de la evolución actual del fenómeno museístico en torno al rol de las colecciones dentro del museo. La globalización, el desarrollo de la tecnología digital, así como la influencia de nuevas corrientes de pensamiento, como el poscolonialismo, 
podrían transformar la manera de definir el museo. Sin embargo, esta transformación forma parte de una evolución más global, que ha estado ocurriendo desde la década de 1970. El autor analiza los principales hitos que han llevado a los museos a dar prioridad al público sobre la gestión de las colecciones, pero también el surgimiento de la autonomía progresiva de las reservas dentro del sistema museístico. Este principio le lleva a considerar conjuntamente la evolución de las reservas y la de los espacios de exposición, a la luz de otras tendencias que pueden observarse en el sistema económico mundial.

\section{Entrées d'index}

Mots-clés : collections, réserves autonomes, réserves mutualisées, mondialisation, postcolonial Keywords: collections, off-site storages, shared storages, globalization, postcolonial Palabras clave: colecciones, reservas autónomas, reservas compartidas, globalización, postcolonial

\section{Notes de la rédaction}

Manuscrit reçu le 15 avril 2020

Version révisée reçue le 15 juin 2020

Article accepté pour publication le 20 juin 2020

\section{Texte intégral}

1 Le musée, pour peu que l'on s'intéresse à son histoire, semble intrinsèquement déterminé par la notion de collection : son fonctionnement s'appuie sur un système organisé de bases de données constituées d'objets, essentiellement matériels, et d'inventaires qui leur sont associés. C'est l'exploitation de ces bases de données particulières qui a permis le développement des sciences modernes, de l'ethnologie ou de l'histoire de l'art. L'ensemble des activités qui en découlent s'appuie sur ce principe : il faut qu'il $\mathrm{y}$ ait au moins, quelque part, accumulation et gestion de collections pour faire fonctionner la mécanique muséale. Ce mode de fonctionnement du musée, particulièrement développé dans le monde occidental, est actuellement réparti sur tous les continents (Mairesse, 2019). C'est évidemment dans cette perspective que la collection a longtemps été présentée aux yeux du grand public comme constitutive du musée, en témoigne la définition qu'en donne le Larousse en ligne : "Lieu, édifice où sont réunies, en vue de leur conservation et de leur présentation au public, des collections d'œuvres d'art, de biens culturels, scientifiques ou techniques ${ }^{1}$. »

2 La définition actuelle du musée par l'ICOM évoque cinq fonctions (il acquiert, conserve, étudie, expose et transmet) ; les muséologues rassemblent cependant plus généralement ses activités en trois domaines : préservation, recherche et communication (Rivière, 1989; Stránský, 1995 ; Mensch, 2020). On a cependant pu observer, ces dernières années, un grand nombre de questionnements autour de la nature même du musée, celle-ci s'éloignant de plus en plus du principe d'accumulation inhérent à son activité. Ainsi, au Japon ou au Brésil, un certain nombre d'établissements se présentent comme des musées mais n'abritent pas de collections (Morishita, 2010 ; OEI \& Ibram, 2016). Ces établissements développent essentiellement des activités d'exposition ou des événements en lien avec le patrimoine, ou avec des thématiques sociétales ${ }^{2}$.

3 Les musées ont-ils encore besoin des objets rassemblés dans leurs collections pour mener à bien leurs activités ? Cet article a pour objet l'étude des conséquences possibles de l'évolution actuelle du phénomène muséal sur la place des collections au sein du musée. La mondialisation, le développement du numérique, ainsi que l'influence de nouveaux courants de pensée, comme le postcolonialisme, pourraient en effet transformer radicalement la manière de définir le musée. Je voudrais émettre l'hypothèse que cette transformation s'inscrit dans une évolution plus globale, qui s'est poursuivie depuis les années 1970. Dans un premier temps, je présenterai les principaux jalons qui ont conduit à privilégier le public sur la gestion des collections. J'étudierai dans un second temps 
l'émergence d'un phénomène moins évoqué dans ce débat, à savoir l'autonomie progressive des réserves au sein du système muséal. Je me fonderai ici essentiellement sur des exemples français, tout en évoquant un certain nombre de situations dans d'autres régions du monde, afin de développer mon propos. Ce principe d'autonomisation des réserves me conduit à envisager de manière conjointe l'évolution des réserves et celle des lieux d'exposition, à la lumière d'autres tendances que l'on peut observer au sein du système économique mondial.

\section{Le public et l'exposition au cœur du musée}

Qu'ils soient de science ou de beaux-arts, les musées sont d'abord présentés comme des lieux de rassemblement, de préservation, d'étude et de conservation des collections. On retrouve ce principe formulé sans ambiguïté dans la première définition du musée par l'ICOM lors de sa fondation en 1946 :

«Le mot musée désigne toutes les collections de documents artistiques, techniques,
scientifiques, historiques ou archéologiques ouvertes au public, y compris les jardins
zoologiques et botaniques, mais à l'exclusion des bibliothèques, exception faite de
celles qui entretiennent en permanence des salles d'exposition3. »

5 Cette muséologie de l'objet, pour reprendre une formule de Peter van Mensch (2020), constitue le principe sur lequel s'appuie le travail scientifique. S'ils privilégient davantage la recherche, les propos de Zbynĕk Stránský (1995) ou de Georges Henri Rivière (1989) confirment le rôle prééminent de la collection. Le musée se présente, dans sa définition par l'ICOM en 1974 (qui perdure jusqu'en 2007), comme une institution " qui fait des recherches sur les témoins matériels de l'homme et de son environnement ». C'est dans ce contexte, pourtant centré sur la collection, qu'émerge la nouvelle muséologie, portée notamment par Hugues de Varine. On sait l'importance de ce mouvement qui place l'homme au cœur du dispositif muséal et dont l'influence, notamment en France, va contribuer à relativiser le rôle joué par les collections en repositionnant les priorités muséales du côté des visiteurs ou des membres de la communauté :

«Car l'écomusée est l'instrument privilégié du développement communautaire. Il ne vise pas d'abord à la connaissance et à la mise en valeur d'un patrimoine [...]. En cela, il ne peut s'identifier au musée traditionnel et leurs définitions respectives ne peuvent pas concorder » (Varine, 1992 : 458).

S’il ne peut s'identifier au musée traditionnel, s'agit-il encore de musée ? C'est dans un premier temps la conclusion qu'en tire le directeur des Musées de France, Jean Chatelain, refusant de reconnaître et de financer ce nouveau modèle (Debary, 2002).

7 Ce moment de relativisation du rôle de la collection se poursuit tout au long des années 1980, non seulement dans le cadre de la nouvelle muséologie, mais de manière plus générale, au gré de la "révolution des publics » et de la transformation progressive du musée en tant qu'espace social, selon une logique médiatique de plus en plus affirmée (Davallon, 1992). Cette dimension sociale du musée - le rôle de démocratisation, mais aussi d'inclusion, d'émancipation, de participation, etc. - se renforce au cours du xxie siècle, notamment dans le sillage de la crise économique de 2007, conduisant les musées à recentrer leurs activités à partir de cet axe (Black, 2012 ; Museums Association, 2012 ; Brown \& Mairesse, 2018)4 . Au rôle social du musée viennent par ailleurs s'ajouter les influences de trois autres facteurs : économique, technologique et postcolonial.

8 La logique économique semble en effet avoir également joué un double rôle à cet égard. Le phénomène le plus spectaculaire est lié aux opérations - peu nombreuses, mais très largement relayées par les médias - de filialisation de certains grands musées, à l'instar du 
Guggenheim de Bilbao, inauguré en 1997 et rapidement devenu un jalon de l'histoire muséale récente (Tobelem et al., 2014). Si cet établissement se constitue peu à peu en une collection propre, cette dernière demeure embryonnaire, se fondant essentiellement sur le patrimoine de l'institution new-yorkaise et sur l'organisation d'expositions temporaires. Les raisons qui ont conduit à l'implantation d'un tel établissement à Bilbao sont moins éducatives ou patrimoniales qu'économiques et liées au développement urbanistique ou touristique de la région. Ce principe se fonde notamment sur le rôle d'attraction de l'enveloppe architecturale du musée et s'est répercuté à travers le développement de filiales de quelques grandes institutions, comme le Centre Pompidou (Metz, Malaga, Bruxelles, Shanghai) ${ }^{5}$. Il a surtout influencé de manière indirecte la construction d'un très grand nombre de musées, notamment en Asie, privilégiant la qualité de l'enveloppe architecturale à celle des collections (souvent inexistantes) pour attirer le public. Par ailleurs, le raisonnement économique n'est pas non plus sans lien avec le développement d'une réflexion sur l'aliénation (le deaccessioning) et la vente d'une partie des collections. Le phénomène est ancien et différemment implanté à travers le monde suivant deux traditions : l'une latine (France, Italie, Espagne), qui privilégie l'inaliénabilité ; l'autre anglo-saxonne (Grande-Bretagne, Pays-Bas, pays scandinaves), en faveur de l'aliénation (Wijsmuller, 2017). Ce sont essentiellement, dans ce dernier cas, des principes de bonne gestion de la collection qui sont mis en avant, afin de ne conserver que des objets directement en lien avec le domaine d'activité du musée, l'argent dégagé par la vente d'objets doit alors être, selon le code de déontologie de l'ICOM, réinvesti au bénéfice de la collection. Si le musée peut se débarrasser de certaines de ses collections, jusqu'où peut-il s'arrêter?

9 Le développement des technologies numériques a lui aussi transformé notre relation aux collections. La numérisation de ces dernières a permis la constitution de bases de données accessibles à distance. Ces dernières offrent des possibilités de recherche remarquables, tant pour le grand public que pour les chercheurs. Par-delà les questions de recherche ou de médiation, certaines opérations de numérisation d'objets ou de sites ont également permis de créer de nouveaux dispositifs expographiques (jeux, supports multimédias, installations) immersifs et spectaculaires, à l'image des expositions mises en œuvre depuis 2018 à l'Atelier des Lumières, à Paris, présenté (notamment par l'Office du tourisme de Paris) comme un musée, et non comme un centre d'exposition ${ }^{6}$. Ce type d'établissements, apparemment de plus en plus populaires, existe à d'autres endroits dans le monde, notamment en Corée du Sud où il s'est développé, par exemple à Andong ou dans la province du Jeollanam-do, sous la forme de musées sans collections, équipés seulement de dispositifs numériques (Ji, $2018:$ 317-330).

La mondialisation du phénomène muséal semble par ailleurs avoir donné lieu à une volonté de déconstruction et de réinterprétation du modèle occidental fondé sur la collection. La définition publique du musée, telle que la propose le Larousse, convoque deux notions : le lieu et la collection. De cette définition, nombreux sont les établissements qui semblent n'avoir conservé que la première notion, soit le lieu et l'édifice qui lui est associé. On a ainsi pu observer, notamment au Japon, l'émergence de structures qui ne cherchent nullement à développer de collections, mais revendiquent l'appellation de musée (Morishita, 2010). On note un phénomène similaire avec les Pontos de Memória brésiliens (OEI \& Ibram, 2016), des structures légères créées dans le cadre d'une politique d'inclusion sociale fondée sur le patrimoine, conçues sans collection ni volonté de permanence. « D’habitude, les centres culturels sont pour le Sud, les musées pour le Nord. Nous voulions briser cette dichotomie, et montrer qu'un nouveau type de musées était possible », plaide la militante " décoloniale » Françoise Vergès (2016 : 31) chargée de concevoir un musée sans objets à La Réunion. Le modèle du musée occidental fondé sur la collection matérielle, exporté durant le xixe siècle à travers le monde, apparaît ainsi comme potentiellement trop occidental et colonialiste, voire prédateur (Icofom, 2017). Il nécessite dans ce contexte, pour nombre de ses détracteurs, une relecture à l'aune des nouveaux rapports de force se dessinant à travers le monde. 
11 Ces différentes tendances semblent converger dans l'affirmation d'une diminution du rôle des collections au sein du musée, aboutissant dès lors à la transformation de l’institution, davantage tournée vers les publics. Les « nouveaux » musées, même les plus révolutionnaires, n'ont cependant pas pour autant renoncé à s'affirmer sur le champ patrimonial ou celui des connaissances, ni à montrer des objets matériels. Une telle situation semble de prime abord paradoxale, car cette ambition patrimoniale ou scientifique repose toujours sur le principe d'accumulation évoqué au début de cet article. Comment, dès lors, sans collections, le musée pourrait-il fonctionner ? On pourrait bien sûr remarquer que les tendances évoquées plus haut ne portent pas sur tous les musées et qu'un grand nombre d'établissements fonctionnent encore de manière traditionnelle. Un autre phénomène mérite aussi d'être évoqué dans ce contexte, qui semble agir en contrepoint du premier : celui de l'autonomisation de la fonction de préservation.

\section{L'autonomisation des réserves}

Beaucoup de musées à travers le monde conservent encore des collections. Où qu'ils se situent, les problèmes de place qu'ils rencontrent paraissent identiques : comme le rappelait Gaël de Guichen lors d'un débat autour des réserves, se référant à une enquête menée par l'Iccrom, "deux musées sur trois manquent de place, dans un musée sur deux les espaces de rangement sont surencombrés » (ICOM France, $2019: 25)$. Cette tendance à l'augmentation des réserves n'est pas récente ; elle est prise en compte dès les années 1980 dans les projets d'expansion de la plupart des infrastructures muséales existantes. Beaucoup d'établissements ont ainsi cherché à aménager des réserves en leur sein ou dans des bâtiments adjacents, mais un certain nombre de grandes institutions, confrontées au manque de place, ont opté pour la construction de centres autonomes, parfois établis à plusieurs dizaines de kilomètres de leur lieu principal. Ainsi, tandis que trois grands musées londoniens (le British Museum, le Victoria \& Albert Museum et le Science Museum) bénéficiaient d'espaces au cour de Londres, les réserves de la Smithsonian Institution étaient installées en dehors de Washington, dans le Maryland, tandis que celles du Musée des sciences de Tokyo déménageaient à près de 80 kilomètres de la capitale7. En France, le premier musée national ayant délocalisé ses réserves est le Musée des arts et métiers. Celui-ci a ainsi, dans le cadre de sa rénovation en 1994, bâti un nouvel espace parfaitement équipé à Saint-Denis (tableau 1). Autant de structures de plus en plus autonomes, présentées en tant que " centre de support ", " centre de collections » ou « centre de conservation et d'étude », bénéficiant du personnel et des infrastructures nécessaires pour l'étude et le stockage des objets, mais aussi pour l'accueil du public (le plus souvent de manière limitée et encadrée). À l'opposé, les plans de rénovation du Grand Louvre prévoyaient la constitution de réserves sur place (qui se sont avérées inondables en cas de crue de la Seine et ont conduit à la construction de réserves à Liévin).

Tableau 1. Principales réserves organisées ou gérées en France soit de manière autonome, soit dans le cadre d'une politique de mutualisation ${ }^{8}$.

\begin{tabular}{|l|l|l|l|}
\hline Réserves autonomes & Année & Réserves mutualisées & Année \\
\hline Réserve du Musée des arts et métiers & 1994 & Musées de Saint-Étienne & 2001 \\
\hline $\begin{array}{l}\text { Chantier des collections du Musée du quai } \\
\text { Branly - Jacques Chirac }\end{array}$ & 1999 & $\begin{array}{l}\text { Musées de la Ville de } \\
\text { Marseille }\end{array}$ & 2004 \\
\hline Rapport du Sénat (Richert) sur les réserves & 2002 & Musées de la Ville de Paris & 2009 \\
\hline $\begin{array}{l}\text { Réserves délocalisées du Musée de l'armée, } \\
\text { Versailles }\end{array}$ & 2002 & $\begin{array}{l}\text { Réserve muséographique } \\
\text { départementale de Quimper }\end{array}$ & 2011 \\
\hline & & &
\end{tabular}




\begin{tabular}{|c|c|c|c|}
\hline $\begin{array}{l}\text { Centre de conservation et d'étude des } \\
\text { collections, Musée des confluences }\end{array}$ & 2002 & $\begin{array}{l}\text { Musées de la Ville de Nancy } \\
\text { et du Grand Nancy }\end{array}$ & 2013 \\
\hline $\begin{array}{l}\text { Centre externalisé de conservation, d'étude et } \\
\text { de restauration du Musée de Valence }\end{array}$ & 2007 & $\begin{array}{l}\text { Réserves mutualisées de la } \\
\text { Ville de Tours }\end{array}$ & 2015 \\
\hline $\begin{array}{l}\text { Réserves externalisées du Musée des beaux- } \\
\text { arts d'Agen }\end{array}$ & 2007 & $\begin{array}{l}\text { Réserves mutualisées de la } \\
\text { Ville de Reims }\end{array}$ & 2020 \\
\hline $\begin{array}{l}\text { Réserves externalisées du Musée Ingres, } \\
\text { Montauban }\end{array}$ & 2008 & $\begin{array}{l}\text { Pôle d'étude et de } \\
\text { conservation des musées de } \\
\text { Strasbourg }\end{array}$ & 2021 \\
\hline $\begin{array}{l}\text { Définition des Centres de conservation et } \\
\text { d'étude (CCE) par le ministère de la Culture }\end{array}$ & 2008 & & \\
\hline $\begin{array}{l}\text { Centre d'étude, de restauration et de } \\
\text { conservation des œuvres (Cerco), Museon } \\
\text { Arlaten }\end{array}$ & 2009 & & \\
\hline $\begin{array}{l}\text { Centre de conservation et d'étude de Lons-le- } \\
\text { Saunier }\end{array}$ & 2010 & & \\
\hline Réserves externalisées du Musée de Dijon & 2010 & & \\
\hline $\begin{array}{l}\text { Réserves du Musée d'art et d'archéologie du } \\
\text { Périgord, Périgueux }\end{array}$ & 2012 & & \\
\hline Centre de conservation et d'étude du Mucem & 2013 & & \\
\hline $\begin{array}{l}\text { Rapport du Sénat (Attard) sur la gestion des } \\
\text { réserves }\end{array}$ & 2014 & & \\
\hline $\begin{array}{l}\text { Centre de conservation du Musée du Louvre, } \\
\text { Liévin }\end{array}$ & 2019 & & \\
\hline $\begin{array}{l}\text { Centre Pompidou francilien - Fabrique de l'art } \\
\text { (Massy) }\end{array}$ & $(2025)$ & & \\
\hline
\end{tabular}

Nombre de réserves externalisées ont ainsi progressivement été construites (en France à Valence, Agen, Montauban, Dijon, Arles, etc.), la plupart du temps dans le cadre de projets de rénovation - par exemple le Musée des confluences à Lyon, ou le Mucem à Marseille) ou lors de la mise en place des centres de conservation et d'étude visant à stocker le patrimoine archéologique (Charnier, 2008). Une telle tendance semble se poursuivre à travers le monde, de nombreux musées (Centre Pompidou, British Museum, Victoria \& Albert Museum) faisant état de projets très ambitieux à cet égard (tableau 2). Ce mouvement est aussi parfois conditionné par une volonté de rationalisation des espaces, plusieurs musées ayant progressivement acquis ou loué des réserves au gré de l'évolution de leurs besoins. Ainsi, un audit britannique réalisé pour les 17 musées nationaux faisait état, en 2003, de 81 lieux de stockage différents, recommandant le regroupement de ces infrastructures afin de réaliser des économies d'échelle (Keene, 2007). De tels principes sont mis en œuvre depuis le début des années 2000 par le biais d'un processus de mutualisation des réserves dans plusieurs villes françaises, notamment à Paris, Marseille, Nancy ou Tours.

Tableau 2. Réserves spécifiques de musées étrangers : quelques dispositifs de présentation des réserves visitables par le grand public 9 .

\begin{tabular}{|l|l|l|l|}
\hline Présentation de réserves & Année & $\begin{array}{l}\text { Réserves spécifiques de quelques } \\
\text { grands musées étrangers }\end{array}$ & Année \\
\hline Galerie d'étude, Musée national & 1972 & Blythe House Acquisition, Victoria & 1979 \\
\hline
\end{tabular}




\begin{tabular}{|c|c|c|c|}
\hline $\begin{array}{l}\text { des arts et traditions populaires, } \\
\text { Paris }\end{array}$ & & $\begin{array}{l}\text { \&Albert Museum, British Museum, } \\
\text { Science Museum, Londres }\end{array}$ & \\
\hline $\begin{array}{l}\text { Visible Storage, Museum of } \\
\text { Anthropology at the University of } \\
\text { British Columbia, Vancouver }\end{array}$ & 1976 & $\begin{array}{l}\text { Museum Support Center, Smithsonian } \\
\text { Institution, Suitland, Maryland }\end{array}$ & 1983 \\
\hline $\begin{array}{l}\text { Visible Storage, Henry Luce } \\
\text { Center for the Study of American } \\
\text { Art, Metropolitan Museum of Art, } \\
\text { New York }\end{array}$ & 1988 & $\begin{array}{l}\text { Darwin Center, Natural History Museum, } \\
\text { Londres }\end{array}$ & 2002 \\
\hline $\begin{array}{l}\text { Visible Storage, New York } \\
\text { Historical Society Luce Center }\end{array}$ & 2000 & Schaulager, Münchenstein & 2003 \\
\hline $\begin{array}{l}\text { Visible Storage Study Center, } \\
\text { Brooklyn Museum, New York }\end{array}$ & 2005 & $\begin{array}{l}\text { Centre national de conservation et } \\
\text { d'étude des collections, Musée de la } \\
\text { civilisation / Réserve muséale de la } \\
\text { Capitale-Nationale, Québec }\end{array}$ & 2004 \\
\hline $\begin{array}{l}\text { Réserves visibles, Musée du quai } \\
\text { Branly - Jacques Chirac, Paris }\end{array}$ & 2006 & $\begin{array}{l}\text { National Museum of Nature and } \\
\text { Science, Collection Center, Tsukuba }\end{array}$ & 2006 \\
\hline $\begin{array}{l}\text { Visible Storage, Ceramics } \\
\text { Galleries, Victorian \& Albert } \\
\text { Museum, Londres }\end{array}$ & 2010 & $\begin{array}{l}\text { Centre des collections du Musée } \\
\text { national suisse, Affoltern am Albis }\end{array}$ & 2007 \\
\hline $\begin{array}{l}\text { Visible Storage, Museum aan de } \\
\text { Stroom, Anvers }\end{array}$ & 2011 & $\begin{array}{l}\text { Powerhouse off-site Museum Storage } \\
\text { Facility, Sidney }\end{array}$ & 2007 \\
\hline $\begin{array}{l}\text { Réserves visitables du Louvre- } \\
\text { Lens, Lens }\end{array}$ & 2012 & $\begin{array}{l}\text { National Museum Collection Center, } \\
\text { Edinburgh }\end{array}$ & 2015 \\
\hline $\begin{array}{l}\text { Appartement témoin du Centre de } \\
\text { conservation du Mucem, Marseille }\end{array}$ & 2013 & $\begin{array}{l}\text { Netherlands Collection Center, } \\
\text { Amersfoort }\end{array}$ & 2021 \\
\hline $\begin{array}{l}\text { World Conservation and } \\
\text { Exhibition Center, British Museum, } \\
\text { Londres }\end{array}$ & 2014 & $\begin{array}{l}\text { National Collection Center (Science } \\
\text { museum group), Wiltshire }\end{array}$ & $(2023)$ \\
\hline $\begin{array}{l}\text { Paisley Museum Store, High } \\
\text { Street, Paisley }\end{array}$ & 2017 & $\begin{array}{l}\text { British Museum Storage and Study } \\
\text { center at Thames Valley Science Park, } \\
\text { Reading }\end{array}$ & $(2023)$ \\
\hline $\begin{array}{l}\text { Depot, Museum Boijmans Van } \\
\text { Beuningen, Rotterdam }\end{array}$ & $(2021)$ & V\&A East Project Storage facility & $(2023)$ \\
\hline
\end{tabular}

La conservation des objets en réserve a donné lieu, parallèlement à ce mouvement d'autonomie, à un questionnement sur l'accessibilité des collections. Si ces dernières sont la plupart du temps disponibles pour les chercheurs, la question de l'accueil du public dans de tels espaces s'est assez rapidement posée. Plusieurs solutions ont progressivement émergé au cours des années 1970. L’une des premières à avoir été mises en œuvre a été conçue par Georges Henri Rivière (1989) pour les collections du Musée national des arts et traditions populaires, par le biais d'une galerie d'étude (en sous-sol) ouverte au public en 1972. La volonté de montrer à la fois des séries d'objets, tout en proposant un regard des " coulisses ", a conduit à la création de plusieurs réserves visitables, notamment à Vancouver, New York et Londres (tableau 2). L'évolution de ces lieux a permis l'émergence de nouvelles solutions visant surtout à évoquer l'univers des réserves plutôt que de promouvoir l'étude systématique, comme au Museum aan de Stroom, à Anvers. C'est dans une telle perspective que plusieurs musées français récents (quai Branly - Jacques Chirac, Louvre-Lens) ont intégré des réserves visibles, sinon visitables, afin d'évoquer la fonction de préservation du musée. La mise en valeur de ces activités a ainsi conduit à la 
constitution, à l'intérieur de centres de recherche comme le Darwin Center de Londres, ou de centres de préservation comme celui du Mucem, de véritables dispositifs d'interprétation des activités de préservation ou de recherche. Certaines formules hybrides, comme le Schaulager, près de Bâle, ou le Paisley Museum Store, se présentent à la fois comme des lieux de conservation de l'art, accessibles à un public spécialisé, et comme une nouvelle manière d'appréhender les collections ; c'est dans une perspective similaire que plusieurs projets récents de réserves, comme le « Depot » du Museum Boijmans Van Beuningen de Rotterdam, ont été mis en œuvre ${ }^{10}$.

L'autonomie des réserves, si elle s'insère dans une logique de rationalisation, a permis de dégager dans les musées de nouveaux espaces pour les expositions et l'accueil des publics, tout en conduisant à un double phénomène. D'une part, les musées apparaissent de plus en plus comme des espaces d'exposition et non de conservation ou de recherche ; d'autre part, ces mêmes espaces dédiés à la préservation et à l'étude du patrimoine acquièrent une réelle autonomie, au point de pouvoir être pensés de manière indépendante. Certes, la plupart des centres de conservation ou d'étude appartiennent encore, administrativement, à la même entité juridique. En revanche, d'un point de vue organisationnel, le bâtiment intitulé "musée ", qui naguère en abritait toutes les fonctions, se voit de plus en plus souvent destiné aux seuls espaces d'exposition. Beaucoup de musées occidentaux fonctionnent ainsi déjà, d'une certaine manière, comme des centres d'exposition sans collection, leur organisation dépendant d'une logistique plus ou moins sophistiquée entre le lieu d'exposition et les espaces réservés aux collections.

\section{Management et gestion des stocks}

Cette situation n'est pas sans rappeler le fonctionnement des organisations industrielles régies à l'aune du management moderne, porté par la spécialisation des tâches et la gestion des flux. Cette organisation a conduit, à l'heure de la mondialisation, à une interdépendance généralisée entre les différents pays producteurs. La diminution des stocks permet des économies considérables, pour autant qu'elle repose sur une logistique suffisante afin d'acheminer " juste à temps » les matières premières ou les produits et services intervenant dans le processus de fabrication (la crise du Covid-19 en a montré la fragilité et les limites). Les règles de la nouvelle gestion publique, fondées sur le management des entreprises (Chong, 2010), dérivent de ces préceptes, impliquant spécialisation et mutualisation, y compris dans le domaine de la culture (Deniau, 2014).

Dans cette perspective, la notion de stock (non productif) peut apparaître comme une aberration économique, puisque sa gestion se révèle très onéreuse (Lord et al., 1989). Le raisonnement économique suppose, lorsqu'il est appliqué de manière stricte aux musées (Grampp, 1989), la diminution maximale des réserves et la vente d'une partie des collections. C'est à un tel raisonnement qu'était notamment arrivé le rapport Jouyet-Lévy (2006) ayant contribué à l'émergence d'un projet de loi en faveur de l'aliénation des collections françaises. Dans le cas où les collections ne peuvent être aliénées, celles-ci doivent néanmoins prouver leur utilité, montrer qu'elles sont étudiées, prêtées, exposées, etc. (Gardner, 2007 ; Pettersson et al., 2010). C'est ce principe qui a favorisé l'accessibilité et l'interprétation des réserves au public, alors qu'elles étaient encore, il y a peu, destinées aux seuls chercheurs et « liées à leur intimité » (Ames, 1986 : 66).

L'émergence d'organisations autonomes chargées de conserver des objets, tandis que d'autres les utilisent, ne constitue pas un phénomène original au sein de notre société. Sur le plan patrimonial, cette solution a été utilisée depuis l'Ancien Régime pour une institution spécifique, le Garde-Meuble de la Couronne, chargé de la gestion du mobilier et des objets d'art des demeures royales. Fondé sur des principes d'inventaire et de conservation, mais aussi de production de mobilier, il s'est progressivement doublé d'une fonction d'exposition (lors de son installation dans ce qui deviendra l'hôtel de la Marine) (Castelluccio, 2002). La constitution de dépôts de stockage accompagne également les 
saisies révolutionnaires ; de tels lieux n'ont pas pour objet la visite du public, même si le dépôt des Petits-Augustins se transforme pour devenir le Musée des monuments français (Bresc-Bautier \& Chancel-Bardelot, 2016). La notion de garde-meuble s'est très largement poursuivie jusqu'à nos jours, à travers le Mobilier national, bien sûr, mais aussi par le biais de nombreuses sociétés commerciales (à destination des entreprises ou des particuliers).

Sur le plan plus spécifiquement artistique, le marché de l'art repose également de plus en plus sur des intermédiaires qui offrent leurs services d'entreposage aux collectionneurs ne disposant pas d'espaces de conservation suffisamment vastes ou adaptés. De nombreux galeristes dépourvus d'espaces de vente classiques ont également opté pour ces solutions nouvelles. Plusieurs types d'intermédiaires ont ainsi développé ce type de services : d'une part des sociétés de transport ou d'emballage spécialisées dans le domaine de l'art et munies d'espaces de stockage (Chenue, LP Art) ; d'autre part des sociétés auparavant spécialisées dans la gestion des entrepôts comme les ports francs (Azimi, 2019). Présentés comme « le plus grand musée d'art de la planète » (Dupuis \& Thiolay, 2016), les Ports francs et entrepôts de Genève (PFEG) accueillent ainsi une clientèle composée de galeristes et de collectionneurs échangeant leurs œuvres dans des locaux aux normes de conservation préventive et de sécurité irréprochables (il en existe de nombreux autres, notamment à Luxembourg ou à Singapour). Si la gestion de certains de ces établissements a pu défrayer la chronique en raison de leur opacité fiscale (ibid. ; Vazzoler, 2019), les ports francs, comme un certain nombre d'entrepôts situés aux portes des grandes métropoles, non loin des aéroports, apparaissent de nos jours comme l'une des solutions optimales en matière de gestion des collections patrimoniales. Dans cette perspective, la réserve muséale autonome, elle aussi logistiquement bien adaptée aux circuits d'échange nationaux et internationaux, se profile comme le pendant non commercial des gardemeubles privés. Elle induit en quelque sorte la possibilité de passerelles entre les deux types d'établissements : " Depot », la nouvelle réserve visitable du musée Boijmans Van Beuningen de Rotterdam, proposera ainsi la location de $10 \%$ de ses espaces à des collectionneurs privés (Quotidien de l'art, 2016).

\section{L'éclatement du musée}

20 Il y a quelque chose de paradoxal dans le monde des musées : celui-ci, qui reposait depuis des siècles sur un principe de base - l'accumulation -, semble apparemment s'en détacher pour privilégier des enjeux sociaux ou de médiation. Une telle tendance, observable au sein d'un certain nombre de musées à travers le monde, notamment en Amérique latine ou en Asie, est influencée par une vision parfois résolument différente du patrimoine, voire dans une perspective anticoloniale, mais aussi pour des motifs technologiques ou économiques. Rien n'indique cependant que le système de valeurs sur lequel se fonde notre société ait soudainement rejeté les principes d'accumulation ; le nombre d'objets acquis ou conservés par de nombreux musées n'a pas réellement diminué, et ces établissements continuent d'être largement confrontés à des problèmes de gestion de leurs collections, dans des réserves souvent trop exiguës.

21 Si la majorité des musées continue de fonctionner en articulant des activités de préservation, de recherche et de communication, un certain nombre d'entre eux ont donc activement cherché à évacuer la fonction de conservation, pour ne retenir que celle de communication avec les publics. Cette tendance peut être interprétée comme la suite inéluctable d'une évolution, d'abord marquée par l'abandon du lien unissant le monde des musées à celui de la recherche, tel qu'il pouvait être encore observé au cours des années 1970 (Rivière, 1989), puis par l'ouverture aux publics. Parallèlement à cette évolution, la gestion de nombreuses réserves a été confiée à des centres de plus en plus autonomes, quoiqu'encore majoritairement rattachés aux musées eux-mêmes, ou à des consortiums d'établissements. Ce mouvement d'autonomisation des fonctions, précédemment réunies 
au sein du système muséal, constitue un phénomène dont l'évolution mérite d'être étudiée avec attention.

D'un point de vue théorique, il n'est en effet absolument pas nécessaire que les fonctions de préservation, de recherche et de communication soient opérées au sein d'un même lieu. En cela, l'évolution du musée ne diffère pas fondamentalement de celle des autres secteurs de production dont la logique repose sur la spécialisation des fournisseurs, le recours à la sous-traitance et à un système de logistique pour assurer ces différents services. On peut observer ce phénomène au niveau de l'ensemble du secteur de la culture, qu'il s'agisse des industries culturelles (cinéma, télévision) ou des arts de la scène (musique, théâtre). Ces secteurs ont connu une évolution similaire : les grands établissements naguère à la tête de troupes d'acteurs, de musiciens, de réalisateurs, de décorateurs, de techniciens, etc. se sont progressivement départis de leur personnel et ont opté pour la sous-traitance. L'organisation des musées les plus récents (le Mucem, le Louvre-Lens), fondée sur la contractualisation de nombreux services (entretien, surveillance, médiation, scénographie), suit en ce sens un schéma identique. Comment éviter, dans ce contexte, que certains établissements dans le monde se revendiquent en tant que musées tout en ne conservant que les aspects les plus visibles de l'institution (architecture et exposition) pour en sous-traiter les autres?

Le système muséal, dans cette perspective, continue donc de fonctionner comme il le faisait il y a deux siècles, mais à partir d'une logique de spécialisation plus complexe, au même titre que la plupart des autres secteurs d'activité. Qu'il s'agisse de musées vides (au Japon) ou de " points de mémoire » (au Brésil), les établissements évoqués dans cet article reposent, à l'instar des centres de collection ou des centres de conservation et d'étude, sur une logique similaire à celle de n'importe quel musée. Le champ muséal, en revanche, se révèle plus hétérogène, étant composé d'entités de plus en plus spécialisées et différentes, bien que toujours plus interdépendantes.

$24 \quad$ S'il semble aisé de délimiter le champ muséal à partir des fonctions qui le composent, la question de la définition du musée, en revanche, apparaît bel et bien comme problématique. À l’intérieur du champ, un certain nombre d'acteurs spécialisés dans l'une des fonctions du système muséal ne sont plus, au vu des définitions classiques, considérés comme des musées... à moins que l'on ne décide de changer les conventions. Depuis son origine dans l'Antiquité, le mot " musée » a connu plusieurs acceptions - temple des muses, cabinet d'étude, assemblée savante, etc. Il n'est pas impossible qu'il évolue pour désigner un centre d'exposition ou de médiation n'abritant plus de collections. Il conviendrait alors d'appeler autrement le champ dans lequel ces centres évoluent, qui devraient néanmoins toujours être associés à d'autres centres de recherche, et d'accumulation d'objets et de données, pour fonctionner selon les principes décrits dans cet article.

\section{Bibliographie}

Ames (Michael M.). 1986. Museums, the Public and Anthropology: A Study in Anthropology of Anthropology. Vancouver : UBC Press.

Azimi (Roxana). 2019. " Le casse-tête du stockage d'œuvres ». L'Hebdo du Quotidien de l'Art, 1697, 5 avril 2019, p. 10-11.

Black (Graham). 2012. Transforming Museums in the Twenty-first Century. Londres : Routledge. DOI : $10.4324 / 9780203150061$

Botte (Julie), Doyen (Audrey) \& Uzlyte (Lina). 2017. " Ceci n'est pas un musée : panorama géographique et historique des définitions du musée ", p. 17-49 in Définir le musée du xхı siècle / sous la direction de François Mairesse. Paris : ICOM/Icofom.

Bresc-Bautier (Genevière) \& Chancel-Bardelot (Béatrice de) (dir.). 2016. Un musée révolutionnaire. Le Musée des monuments français d'Alexandre Lenoir. Paris : Hazan.

Brown (Karen) \& Mairesse (François). 2018. "The definition of the museum through its social role ». Curator: The Museums Journal, 61(4), p. 525-539. 
Castelluccio (Stéphane). 2002. Les Collections royales d'objets d'art, de François Ier à la Révolution. Paris : Les Éditions de l'Amateur.

Charnier (Jean-François). 2008. "Les centres de conservation et d'étude ». Les Nouvelles de l'archéologie, 113, en ligne : http://journals.openedition.org/nda/572 [consulté le 23 novembre 2020].

DOI : $10.4000 /$ nda.572

Chong (Derrik). 2010. Arts Management. Londres: Routledge [2e édition]. DOI : 10.4324/9780203995358

Davallon (Jean). 1992. "Le musée est-il vraiment un média ? ». Publics et Musées, 2, p. 99-123, en ligne : https://www.persee.fr/doc/pumus_1164-5385_1992_num_2_1_1017 [consulté le 23 novembre 2020].

DOI : 10.3406/pumus.1992.1017

Debary (Octave). 2002. La Fin du Creusot ou l'art d'accommoder les restes. Paris : Éditions du CTHS (Le regard de l'ethnologue, 13).

Deniau (Marie). 2014. Étude exploratoire sur les nouvelles pratiques de mutualisation ou de coopération inter-organisationnelles dans le secteur culturel. Paris, ministère de la Culture et de la Communication, Département des études, de la prospective et des statistiques, 16 juillet, en ligne : https://www.culture.gouv.fr/Sites-thematiques/Etudes-et-statistiques/Publications2/Rapports-derecherche/Nouvelles-pratiques-de-mutualisation-et-de-cooperation-dans-le-secteur-culturel [consulté le 23 novembre 2020].

Dupuis (Jérôme) \& Thiolay (Boris). 2016. « Ports francs de Genève, un gigantesque garde-meubles du marché de l'art ». L'Express, 18 mars 2016, en ligne : https://www.lexpress.fr/culture/art/portsfrancs-de-geneve-un-gigantesque-garde-meubles-du-marche-de-l-art_1773875.html [consulté le 23 novembre 2020].

Dupuy (Leslie). 2002. «Le Musée d'art et d'industrie de Saint-Étienne : le renouveau d'un musée de société industrielle ». La Lettre de l'OCIM, 82, p. 23-29.

Ferriot (Dominique) \& Giuliana-Peyrard (Nathalie). 2015. Arts et Métiers. Chronique d'une rénovation. Paris : Musée des arts et métiers / CNAM.

Gardner (Laura). 2007. "The uses of stored collections, in some London museums ». Papers from the Institute of Archaeology, numéro spécial, p. 36-78.

DOI : $10.5334 / 294$

Gérard (Pierre-Antoine) \& Delaunay (Sandra). 2014. « Des réserves mutualisées et externalisées : un élan pour les opérations de récolement? ». La Lettre de l'OCIM, 153, p. 47-52.

Grampp (William D.). 1989. Pricing the Priceless: Art, Artists and Economics. New York : Basic Books Inc.

Icofom. 2017. Icofom Study Series, 45, « The predatory museum ».

ICOM France. 2019. Les réserves sont-elles le coeur des musées ? Synthèse de la soirée-débat du 18 avril 2019 (auditorium Colbert, Paris). Paris : ICOM France.

INP [Institut national du patrimoine]. 2014. Les Réserves : Pour une gestion optimale des collections. Dossier de formation permanente. Paris : Institut national du patrimoine, Centre de ressources documentaire du Département des conservateurs. En ligne : http://mediathequenumerique.inp.fr/Dossiers-de-formation/Reserves-pour-une-gestion-optimale-des-collections [consulté le 23 novembre 2020].

Ji (Young Ho). 2018. La Numérisation du patrimoine culturel au sein des musées coréens : Une approche de la médiation numérique des institutions muséales. Thèse soutenue le 15 janvier à l'Université Sorbonne Nouvelle - Paris 3.

Jouyet (Jean-Pierre) \& Lévy (Maurice). 2006. L'Économie de l’immatériel. La croissance de demain. Rapport de la Commission sur l'économie de l'immatériel. Paris : La Documentation française.

Kazerouni (Alexandre). 2017. Le Miroir des cheikhs. Musée et politique dans les principautés du golfe Persique. Paris : PUF (Proche-Orient).

Keene (Suzanne). 2007. " Collections in the English national museums: the numbers ». Papers from the Institute of Archaeology, numéro spécial, p. 115-134.

DOI : $10.5334 / 297$

Labourdette (Marie-Christine). 2015. Les Musées de France. Paris : PUF (Que sais-je ?).

Lord (Barry), Lord (Gail Dexter) \& Nicks (John). 1989. The Cost of Collecting. Londres : HMSO.

Maczek (Ewa) (dir.). 2019. "Vers une nouvelle définition du musée ? ». La Lettre de l'OCIM, 186, p. 10-27.

Mairesse (François). 2015. « L'économique et/ou le social ? Quel rôle futur pour le musée ? ", p. 117143 in L'Inclusion sociale. Les enjeux de la culture et de l'éducation / sous la direction de Anne 
Barrère et François Mairesse. Paris : L’Harmattan.

Mairesse (François). 2019. « Le principe d'accumulation », p. 203-216 in Musées, Mutations... / sous la direction de Joëlle Le Marec, Bernard Schiele et Jason Luckerhoff. Dijon : Éditions universitaires de Dijon (OCIM).

May (Roland). 2011. " Regard sur la conservation préventive dans les musées de France ». La Lettre de l'OCIM, 138, p. 5-10.

DOI : $10.4000 /$ ocim.973

Mensch (Peter van). 2020. Vers une méthodologie de la muséologie. Traduit de l'anglais par Chloé Kuhn. Préface de François Mairesse. Paris : L’Harmattan (Muséologies).

Morishita (Masaaki). 2010. The Empty Museum. Western Cultures and the Artistic Field in Modern Japan. Farnham : Ashgate.

Museums Association. 2012. Museums 2020 Discussion Paper. Londres : Museums Association. En ligne : https://www.museumsassociation.org/app/uploads/2020/o6/24072012-discussionpaper.pdf [consulté le 23 novembre 2020].

OEI [Organização dos Estados Ibero-americanos] \& Ibram [Instituto Brasileiro de Museus]. 2016. Memory Spots: Methodology and Practices in Social Museology. Brasília : OEI/Ibram.

Pettersson (Susan), Hagedorn-Saupe (Monika), Jyrkkiö (Teijamari) \& Weij (Astrid) (dir.). 2010. Encouraging collections mobility - A way Forward for Museums in Europe. Helsinki : Finnish National Gallery, Amsterdam : Erfgoed Nederland, Berlin : Staatliche Museen.

Quotidien de l'art. 2016. " Le Museum Boijmans van Beuningen propose ses réserves aux collectionneurs privés ». Le Quotidien de l'art, 1003, 16 février 2016, p. 4.

Tobelem (Jean-Michel), Lus Arana (Luis Miguel) \& Ockman (Joan). 2014. Les Bulles de Bilbao. Paris : Éditions B2 (Actualités).

Rivière (Georges Henri). 1989. La Muséologie selon Georges Henri Rivière. Cours de muséologie, textes et témoignages. Sous la direction d'Hélène Weis. Paris : Dunod.

Simonnot (Nathalie) \& Lheureux (Rosine) (dir.). 2018. Architectures et espaces de la conservation, 1995-2015. Archives, bibliothèques, musées. Villeneuve-d'Ascq : Presses universitaires du Septentrion (Architecture et urbanisme).

Stránský (Zbynĕk Z.). 1995. Muséologie : Introduction aux études. Brno : Université Masaryk.

Varine (Hugues de). 1992. "L'écomusée », p. 446-487 in Vagues. Une anthologie de la nouvelle muséologie. Vol. 1 / sous la direction de André Desvallées. Mâcon : Éditions W, Savigny-le-Temple : MNES [1're parution : 1978, La Gazette (Association canadienne des musées), 11, p. 28-40].

Vazzoler (Marine). 2019. "Les ports francs, vers plus de transparence ? ». L'Hebdo du Quotidien de l'Art, 1697, 5 avril 2019, p. 12-13.

Vergès (Françoise). 2016. "A museum without objects », p. 25-38 in The Postcolonial Museum. The Arts of Memory and the Pressures of History / sous la direction de Iain Chambers, Alessandra De Angelis, Celeste Ianniciello, Mariangela Orabona et Michaela Quadraro. Londres : Routledge.

Wijsmuller (Dieuwertje). 2017. Deaccessioning \& Disposal in Europe 20o8-2017. Amsterdam : Mondrian Fund.

\section{Notes}

1 Dictionnaire de français : www.larousse.fr/dictionnaires/francais/musée/53378 (consulté le 12 novembre 2020).

2 La proposition de définition évoquée à Kyoto en 2019, qui a tant agité la communauté muséale, évacuait largement le principe de la collection et des fonctions qui lui sont associées au bénéfice d'un rôle plus actif, voire activiste, du musée (respect des droits de l'homme, écoute des minorités, justice sociale, développement durable, etc.) au sein de la société ou des communautés qui la composent (Maczek, 2019).

3 Statuts de l'ICOM, 1946, art. 2, section II. Cité par Botte et al., 2017.

4 On peut observer une corrélation assez forte entre le développement des crises économiques et la mise en valeur du rôle social des musées par ces derniers, ou son apparition dans la littérature muséologique (Mairesse, 2015).

5 Le cas de l'implantation du Louvre à Abou Dhabi diffère quelque peu des logiques présentées ici (Kazerouni, 2017). Il intègre cependant des questions identiques en matière de collections et induit des flux importants d'œuvres entre la France et les Émirats, dans le cadre des politiques de prêts du Louvre et des Musées de France. 
6 Voir : https://www.parisinfo.com/musee-monument-paris/180019/Atelier-des-Lumières. L'Atelier des Lumières est présenté sur Wikipédia comme « un centre d'art numérique »: https://fr.wikipedia.org/wiki/Atelier_des_Lumieres. Il apparaît cependant également dans la catégorie "musée " sur le site de référencement touristique Tripadvisor. Voir : https://www.tripadvisor.fr/Attraction_Review-g187147-d13986330-Reviews-

Atelier_des_Lumieres-Paris_Ile_de_France.html (sites consultés le 19 novembre 2020).

7 Il existe peu de références à cet égard, et la plupart des informations ont été collectées à partir d'Internet. Pour la Smithsonian Institution, voir la factsheet éditée par l'Institution et disponible sur https://www.si.edu/newsdesk/factsheets/museum-support-center (consulté le 19 novembre 2020). Pour les arts et métiers, évoqués ci-après, voir Ferriot \& Giuliana-Peyrard, 2015.

8 Sources principales du tableau : Labourdette, 2015 ; Simonnot \& Lheureux, 2018 ; May, 2011 ; Dupuis, 2002 ; Gérard \& Delaunay, 2014 ; INP, 2014 ; Le Quotidien de l'art (plusieurs numéros depuis 2013).

9 Sources : cf. note 8.

10 Le projet du « Depot » est décrit sur le site du bureau d'architecture MVRDV qui en a la charge : https://www.mvrdv.com/projects/10/depot-boijmans-van-beuningen (consulté le 19 novembre 2020).

\title{
Pour citer cet article
}

Référence papier

François Mairesse, « La collection a-t-elle un avenir au sein du musée ? », Culture \& Musées, 37 | 2021, 31-52.

Référence électronique

François Mairesse, « La collection a-t-elle un avenir au sein du musée ? », Culture \& Musées [En ligne], 37 | 2021, mis en ligne le 01 juin 2021, consulté le 06 juillet 2021. URL :

http://journals.openedition.org/culturemusees/6124 ; DOI :

https://doi.org/10.4000/culturemusees.6124

\section{Auteur}

\section{François Mairesse}

Université Sorbonne Nouvelle, Cerlis, CNRS, ICCA

François Mairesse est muséologue, professeur d'économie de la culture et titulaire de la chaire Unesco sur l'étude de la diversité muséale et son évolution, Museum Prospect, à l'Université Sorbonne Nouvelle - Paris 3 (Cerlis, CNRS, labex ICCA). Il enseigne également la muséologie à l'École du Louvre. Il a auparavant dirigé le Musée royal de Mariemont, en Belgique (de 2002 à 2010), et a présidé le comité international de muséologie de l'ICOM/Icofom. II a publié de nombreux articles et ouvrages dans le domaine de la muséologie, de l'économie de la culture ou de la médiation culturelle, dont les plus récents sont : Zbynĕk Z. Stránský et la muséologie. Une anthologie (dir., L'Harmattan, 2019) ; La Médiation culturelle (avec Bruno Nassim Aboudrar, PUF, 2018) ; Enquête sur les pratiques savantes ordinaires (avec Joëlle Le Marec, Le Bord de l'Eau, 2017) ; et Accueillir et surveiller. Les métiers du gardien de musée (dir., avec Anne Monjaret, La Documentation française, 2017).

Courriel : francois.mairesse[at]sorbonne-nouvelle.fr

Articles du même auteur

Claire Merleau-Ponty. Le Jeune Public au musée [Texte intégral]

Paru dans Culture \& Musées, 35 | 2020

André Gob. De Rome à Paris. Retour sur l'origine du musée moderne [Texte intégral] Paru dans Culture \& Musées, 35 | 2020

Introduction [Texte intégral]

Paru dans Culture \& Musées, 27 | 2016

«Sinon, j'aurais pu devenir directeur de musée, attendre mes 65 ans » [Texte intégral] Entretien avec Harald Szeeman (réalisé les 21 et 28 juillet 1996 par François Mairesse) Paru dans Culture \& Musées, 27 | 2016

\author{
Noémie Drouguet, Le Musée de société : De l'exposition de folklore aux enjeux \\ contemporains [Texte intégral] \\ Paris : Armand Colin, 2015 \\ Paru dans Culture \& Musées, 27 | 2016
}




\section{Droits d'auteur}

Culture \& Musées 